\title{
Representación en la Enseñanza del Patrimonio. Abordaje conceptual y metodológico aplicado en el Campus de Goiabeiras de la Universidade Federal do Espírito Santo, Brasil"
}

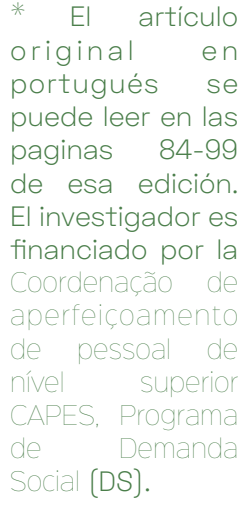

1 Protección y valorización del patrimonio cultural brasileño en el ámbito del desarrollo turistico económico.

$2 \mid 1^{\circ}$ Encuentro de los Intendentes Regionales, Secretarios Estatales en el Área de Cultura, Alcalde de Municípios Interesados, Presidente y Representantes de Instituciones culturales.

3 | Gentilicio de la región de Espírito Santo, Brasil.

\section{Introducción}

Tratado en prácticas de enseñanza académica contemporáneas, el Patrimonio aborda un repertorio que según teóricos paradigmáticos del campo desde su etimología a las prácticas conservacionistas. El desafío a tratar es el avance disciplinar, por medio de la inserción de prácticas contemporáneas trascendentes al enfoque filosófico-arquitectónico acerca del patrimonio y articuladoras del alumno en un contexto interdisciplinar de reconocimiento. La hipótesis es que, adoptando nuevas prácticas pedagógicas integradoras y derivadas de distintos abordajes y temáticas, el estudiante migre de una figura pasiva a una figura activa, actuando en el campo cognitivo, de reconocimiento y de debate. En ese avance metodológico, se levanta la pregunta: ¿es posible aprovechar la capacidad cognitiva y representativa del alumno en el proceso de aprendizaje para la reflexión y práctica de preservación del patrimonio?

En el contexto brasileño, la trayectoria de la enseñanza dirigida al tema del patrimonio tiene su principio en la década de 1970, a partir de una iniciativa del Instituto do Patrimônio Histórico e Artístico Nacional (IPHAN). Asociada a la gestión de Renato Soeiro (1967-1979), la segunda etapa de actuación del Instituto tuvo como énfasis el Patrimonio Urbano, marcada por la afirmación de triple extensión -tipológica, cronológica y geográfica- de bienes patrimoniales (Choay, 2000). Esa ampliación, fomentada por procesos de desarrollo urbanístico, requiere de una protección del patrimonio asociada medidas más amplias, de manera que concilie la conservación y el desarrollo. Por lo tanto, el período está marcado por una revisión de bases científicas de actuación de la institución, expresada en la requisición de ayuda internacional experta, iniciada con la visita técnica de Michel Parent (1966-67), Inspector Jefe de los Monumentos Franceses, y manifiesta en el informe "Proteção e valorização do patrimônio cultural brasileiro no âmbito do desenvolvimento turístico e econômico"' publicado por UNESCO en 1968.

Al mismo tiempo, el IPHAN establece el proceso de descentralización institucional e integración de la política nacional a los estados y municipios, con la finalidad de obtener apoyo en la identificación, tutela y protección de los bienes patrimoniales de importancia regional y local. Tal iniciativa reafirma la necesidad de ampliar el grupo de profesionales habilitados en el campo. Hasta ahora, la formación de técnicos del IPHAN se da, sobre todo, en los sitios de obra. En consonancia, el Compromisso de Brasília de abril de 1970 (IPHAN, 2004, p. 137), elaborado en el 10 Encontro dos governadores de Estado, Secretários Estaduais na Área Cultural, Prefeitos de Municípios Interessados, Presidentes e Representantes de Instituições Culturais², recomienda la formación de arquitectos restauradores, conservadores de pintura, escultura y documentos, archivólogos y museólogos. En respuesta a ello, fue creada la primera carrera nacional de especialización de arquitectos restauradores, vinculada a la Universidade de São Paulo, en 1974. Pensada para ser itinerante, la carrera se dictó en 1976 en la Universidade Federal de Pernambuco, en 1978 en la Universidade Federal de Minas Gerais, y en 1980-81 en la Universidade Federal da Bahia, permaneciendo allí como la sede hasta la actualidad e integrando un programa de postgrado.

Espírito Santo, es precursora en la formación de arquitectos incluyendo el tema patrimonial en el nivel de graduación. Empieza en la carrera de Arquitectura y Urbanismo, perteneciente al Centro de Artes da Universidade Federal do Espírito Santo fue creado en 1978 e instalado en 1979. El debate pasa junto al Patrimônio Histórico, Artístico e Cultural, como asignatura electiva, con 60 créditos hasta el año de 1994 cuando, en el contexto de revisión del proyecto pedagógico, la materia pasa a ser obligatoria, con 90 créditos. En conjunto, en el periodo entre 1991 y 2018, el plan de enseñanza de la asignatura se consolidó en un abordaje apoyado en una carta indicativa enfocada en los siguientes subtópicos: a) Conceptualización del patrimonio cultural, los bienes muebles e inmuebles; b) El patrimonio natural y el urbano; c) Nociones de principios teóricos y legales (levantamientos, conservación, declaratorias y restauración] de sitios naturales, históricos y elementos construidos; d) Las herramientas de preservación de la arquitectura, paisaje natural y edificado; e) Centrarse en el patrimonio arquitectónico y cultural capixaba ${ }^{3}$. 
La asignatura Patrimônio Histórico, Artístico e Cultural tiene como objetivos específicos desarrollar un trabajo reflexivo y empírico sobre la práctica de la preservación, resaltar la complejidad de cuestiones adjuntas, sobre todo a partir de la identificación del conjunto y sus dimensiones -económicas, políticas, sociales, históricas y artísticas-, preparar instrumental metodológico de investigación histórica y caracterización arquitectónica, análisis y propuesta arquitectónica, direccionado para la elaboración de intervenciones proyectuales en estructuras consolidadas. El contenido programático se estructura en cinco etapas, dedicadas i) a las nociones de Monumento y patrimonio; ii) a la política de preservación del patrimonio cultural en Brasil; iii) a la teoría y práctica de conservación y restauración; iv) a la intervención en estructuras consolidadas; v) a la metodología de intervención en estructuras consolidadas.

Con vista a la experimentación proyectual de esos contenidos, además de clases expositivas al mismo tiempo, la disciplina se desarrolla por medio de orientación de dos actividades: 1) Propuesta de Intervención en estructura pre existente, desarrollada en tres etapas: 1.1) Interpretación histórica y caracterización urbano arquitectónica; 1.2) Diagnóstico de daños; 1.3)Intervención de proyecto en nivel de estudio preliminar y 2) Estudio de caso, con descripción e interpretación de intervención urbano paisajística y arquitectónica en pre existencia crítica.

En sintesis, la disciplina se estructura como lugar de enfoque teórico, histórico y proyectual, en la perspectiva de comprensión plegable del tema patrimonial a las de la arquitectura y del urbanismo, al proyecto y la planificación, teniendo como supuestos (Solà-Morales, 2006): 1) el reconocimiento de que los problemas de la intervención en arquitectura histórica son, primera y fundamentalmente, problemas de arquitectura $y$, en este sentido, la lección de arquitectura del pasado es el resultado de un diálogo a partir de la arquitectura del presente y no a partir de posturas defensivas y de preservación; 2) el entendimiento de que el edificio tiene una capacidad de expresión y que los problemas de la intervención en la arquitectura histórica no son problemas abstractos ni problemas que puedan ser formulados de forma definitiva, pero que se presentan como problemas concretos sobre estructuras concretas. Los resultados son trabajos con triple contribución: inventario arquitectónico, diagnóstico de daños y propuesta proyectual. La primera contribución resulta del cumplimiento de vacíos historiográficos sobre la arquitectura en Espirito Santo; el tercer resultado actúa en el reconocimiento del valor potencial de herencias patrimoniales y en el enfrentamiento del proyecto en la planificación.

En su mayoría, los objetos empíricos de las actividades propuestas en la disciplina incluyen la escala del edificio, siempre ampliada para entender y articular el objeto de la actividad con el contexto urbano o rural de inserción, de acuerdo con la triple ampliación del patrimonio identificada por Choay (2000). Las áreas incorporadas a las edificaciones de estudio son plazas, parques, calles, elementos paisajísticos, físico-geográficos. También, en su mayoría, son edificios situados en la región metropolitana de Vitória y de carácter público, con vistas a la necesidad de realización de actividades de levantamiento, como mediciones, registro fotográfico, reconocimiento de patologías.

En el periodo de 2018/2 la disciplina, ofrecida por los autores, propone ampliar la noción del patrimonio a la escala territorial, incluyendo una metodología empírica fomentada por la reflexión sobre la práctica de preservación y utilizando la representación como instrumento interpretativo. En esta perspectiva, la fase analítica lleva la definición de las principales características del territorio a ser potencializados como elementos de valorización territorial (Fanfani et al, 2014).

La propuesta de ampliación conceptual del patrimonio al nivel de patrimonio territorial se basa entonces en la superación de la articulación objeto-contexto de localización, a fin de comprender el proceso de construcción del valor patrimonial, en el espacio y en el tiempo. En este sentido, la ampliación conceptual del patrimonio al nivel territorial se justifica en la ruptura de dos paradigmas: la supresión de la dicotomía conservación y desarrollo, para no crear islas de protección disociadas de un envejecimiento inherente al proceso histórico, aisladas de las áreas donde es permitido el desarrollo y son "sacrificadas" las acciones de conservación así como también la evolución de su entendimiento del objeto al proceso, asimilando el patrimonio como interacción antrópico ambiental, en su diacronía (Poli, 2015).

Esta perspectiva implica una elaboración disciplinaria colectiva, centrando el enfoque en nuevos modos de aprendizaje. Así, el presente artículo presenta la 
experiencia de la asignatura Patrimônio Histórico, Artístico e Cultural, de la clase de 2018/2 de la carrera de Arquitectura y Urbanismo de la Universidade Federal do Espírito

(Brasil) como una propuesta académica centrada en la interpretación del Patrimonio Territorial a partir de un sistema de representaciones.

La representación como potencia analítica se apoya en la metodología de la Escuela Territorial Italiana, cuando propone una representación direccionada a los sedimentos patrimoniales: tipos y padrones, normas de formación y transformación, estructuras y figuras territoriales, vínculos de identidad, etc.

De este modo, la interpretación morfo-tipológica de las relaciones que componen el patrimonio estructuran los Atlas Patrimoniales (Magnaghi, 2017) utilizados como método de análisis. La construcción experimental de los mapa conceptual territorial, pretende en representar los elementos complejos (y describir sus normas de formación y de reproducción histórica) que son considerados activos (valores) patrimoniales (por tanto, bienes comunes) que pueden ser tratados como recursos en los proyectos de transformación, siempre que mantengan o aumenten su valor. Para ese fin, se utiliza el concepto del Estatuto del Territorio (Magnaghi, 2016), que consiste en la descripción, interpretación y representación del patrimonio territorial y sus normas de transformación.

\section{Metodología}

La aproximación conceptual es dada por la incorporación del pensamiento multidisciplinar de la Escuela Territorial Italiana; en especial con el reconocimiento del territorio como patrimonio, entendido de manera procesal (Poli, 2015) y como una construcción social (Magnaghi, 2010). El territorio es comprendido como "una estructura compleja y fuertemente articulada en sus componentes materiales e inmateriales" (Magnaghi, 2005), componentes que son reconocibles en una lectura diacrónica, realizada por medio de la identificación de sucesivos procesos de adición y de sustracción, los actos de territorialización que transforman el espacio en espacio habitado (Santos, 2008). Se trata, por lo tanto, no solamente de una reducción o de una escala geográfica, sino también temporal:

Las diversas escalas de la región geográfica y del lugar singular requieren la lectura de los procesos de formación del territorio en su larga duración, para reinterpretar invariantes, permanencias, sedimentos materiales y cognitivos a los cuales producir nuevos actos de territorialización. (Magnaghi, 2010, p. 62)

El territorio se revela como producto de "una relación entre las entidades vivas, el hombre y la naturaleza, en el largo tiempo de la historia” (Magnaghi, 2010) y, desde está definición, se aprende a reconocer el enfrentamiento de la transformación de las normas genéricas de desarrollo y a utilizar los caracteres de identidad, justificado a partir de los procesos de reconocimiento, como recursos territoriales, dando utilidad al bien, y conectando al contexto local, fundamentalmente (Poli, 2015).

\section{En la metodología territorialista el patrimonio territorial es un sistema de relaciones sinérgicas entre cualidades peculiares del ambiente físico (clima, flora, fauna, geo/hidromorfología, sistemas y neo-ecosistemas ambientales), del entorno construido (permanencias y resistencias urbanas de larga duración. tipos construidos urbanos y territoriales, técnicas, materiales, cualidad de masa territorial, características del entorno). (Magnaghi, 2005, p. 10)}

Para ello, se da prioridad a la producción del conocimiento territorial por medio de la representación, ordenada en descripciones analíticas de capas y de sedimentos del objeto de estudio que, después, son confrontadas en acciones interpretativas. Permiten así, una lectura sintética-descriptiva, donde se evidencian componentes, relaciones, puntos críticos y potencialidades y se identifican los elementos de estructuración del patrimonio territorial estudiado.

El Atlas es destinado a la descripción y a la interpretación de las características
de identidad del territorio, con el objetivo de identificar los modos de operación de
las invariantes estructurales, de evaluar su estado de conservación y establecer
normas de reproductibilidad de las estructuras de identidad. (Carta, 2011) En este sentido, la representación como método dirigido a la construcción del Atlas Patrimonial, es un instrumento no solo documental, sino también interpretativo, ya que destaca variados niveles de información. Las representaciones de los Atlas son articuladas en tres niveles: i) información, donde se obtiene y/o produce una base de datos sobre el territorio de estudio; ii) conocimiento, donde los datos obtenidos 
se comparan y calibran, de acuerdo con objetivos específicos; iii) interpretación, que consiste en la descripción y representación compleja de las identidades territoriales y paisajísticas, a partir de las cuales se moldean las directrices y las decisiones sobre los bienes patrimoniales (Poli, 2012). Por lo tanto, es un aporte analítico-descriptivoproyectual, utilizado de manera metodológica en todas las etapas de la disciplina.

Con la participación activa de los estudiantes, las experiencias de la disciplina son hechas en trabajos de campo, seguidas de representaciones participativas, que tienen la intención de promover diálogos, facilitar debates y discusión en la búsqueda de un proceso más horizontal.

Las articulaciones se dividen en tres etapas: a) análisis, realizado a través de un inventario previo, donde se identifican, clasifican y categorizan los bienes de valor del campus; b) síntesis, donde los mapeos anteriores se confrontan y son identificadas las capas del patrimonio territorial (física, construida y antrópica), que sirven de base para la definición de unidades de paisaje, bajo las cuales se interpretan diacrónicamente los valores y recursos potenciales del patrimonio territorial; c) experiencia proyectual, fundamentada a partir de una interpretación crítica.

El objeto de estudio, Camous Universitario de Goiaberas, Brasil, tiene su selección justificada por ser el primer campus de la Universidade Federal de Espírito Santo, porque tiene un carácter territorial de menor escala geográfica, por tener fácil acceso para los estudiantes y por la diversidad de ambientes (físico, construido y antrópico). Además, por tener una perspectiva histórica reciente y estar inserto en una lógica de desarrollo regulada por la Administración del Campus Universitario y normalizada por el Plan Director Universitario con lo cual promueve una amplia discusión crítica interna. Otra premisa de la selección es la perspectiva de aplicabilidad de una metodología de enseñanza, de utilización apacible y retroalimentación en períodos escolares posteriores.

Aun así, el sentido de lugar emana de experiencias vividas directamente (Bates, 2019), moldeadas de manera consciente o inconsciente a lo largo del tiempo (Bates, 2018; Chawla, 2004). Desde un punto de vista fenomenológico, esto sugiere una conexión persona-lugar a medida que las experiencias son vividas (Gendlin, 2004). Construir conexiones así no solo beneficia el sentido de uno mismo y de los demás, sino que también, promueve un sentido de respeto y dirección para la protección del territorio. (Mannion \& Lynch, 2016). Desde esta perspectiva, la representación del patrimonio territorial de la universidad, desde la visión del estudiante, promueve la potencialización de una relación persona-lugar preexistente, puesto que implica una comunión y confrontación de dos representaciones: una funcional-cualitativa de las características extrínsecas del lugar, difundida a lo largo la carrera y una abstractacognitiva, capaz de identificar características de identidad.

De esa manera, la experiencia ocurre por medio de procesos alternativamente colectivos e individuales, abarcando actividades de análisis, por medio de acciones de valoración, identificación y caracterización. En síntesis, delimita la Unidad de Paisaje (UP) y enumera sus elementos de relevancia y por último, la definición de escenarios estratégico-proyectuales y la indicación de direcciones con prioridad de intervención, relativas a cuatro órdenes de medidas prevalentes: la conservación, la valorización, la recualificación y la transformación.

La primera etapa, el análisis, se subdivide en dos momentos, el colectivo (debates) y el individual (valoración). Se realizan estudios de campo colectivos para obtener material gráfico, cartográfico, histórico, con el fin de respaldar un análisis cognitivo de las características del territorio de estudio: el Campus de Goiabeiras a partir de un análisis global. Tiene como método el pre-inventario, cuyo objetivo es la producción de un mapeo general del Campus de acuerdo con la valoración e identificación y caracterización de los bienes de acuerdo con: denominación, tipo (aislado, grupo), proyecto (autor, fecha, uso actual), situación, categoría de valor, descripción arquitectónica, contextualización geográfica, histórica y urbana, estado de conservación (de lo bien aislado, del grupo arquitectónico, del ambiente), falta de preservación (urgente, a corto plazo, mediano plazo), riesgos potenciales o inminentes, existencia de protecciones, documentación gráfica y cartografía.

En la segunda etapa, de síntesis, se comparan las informaciones obtenidas en la etapa anterior, las mismas son puestas en relación con determinados niveles analíticos para describir las características estructurales del contexto de estudio, destacando la interacción entre los componentes relacionados con la dimensión ambiental y ordenamiento de las informaciones de dimensión antrópica, en su morfotipología, diacronía y funcionamiento (Carta, 2011). 
El procedimiento de mapeo con proyección en cuadro blanco es adoptado en la identificación de la Unidad de Paisaje. Este análisis tiene como producto una hoja que comprende: la delimitación y el mapeo de la Unidad de Paisaje, caracterizada por sus elementos de relevancia, como área construida, caminos, accesos, geomorfología, tendencias de modificación; tambien la contextualización de la unidad a partir de las capas físicas, construidas y antrópicas, identificando la relevancia para la UP y la verificación de las relaciones directas o indirectas con otras unidades.

En la hoja de paisaje, cada lectura de la estructura de larga duración de la invariante va acompañada de la individualización de las dinámicas de transformación, de los valores y de las cuestiones críticas, finalizando como el aparato normativo de las "directrices politicas". (Magnaghi, 2014, p. 116)

La lectura y la delimitación de las unidades de paisaje por parte de los estudiantes y sus abanicos de valores, son cuestiones criticas y dinámicas de transformación que conducen a un pensamiento sobre los escenarios tendenciales de esos ambientes, puede ser identificada o no la devaluación, aprovechamiento o utilización de elementos de valor en favor de cada unidad de paisaje correspondiente.

La representación propone la articulación de las estructuras territoriales para que, a nivel de proyecto, sean definidas invariantes, refiriéndose a la identidad de larga duración en los procesos de formación del territorio de estudio, figuras territoriales, reconocibles por sus especificidades y un estatuto normativo donde se definan estrategias de actuación con miras a crear valor territorial agregado (Gisotti, 2016; Magnaghi, 2016).

Así, y finalmente, en la última etapa, se definen los escenarios estratégicoproyectuales, en los que se resumen todos los datos recolectados en una única estructura analítico-interpretativa, por medio de la elaboración del partido arquitectónico. Esto comienza con la indicación de las direcciones con prioridad de intervención, en referencia a cuatro órdenes de medidas predominantes; en ellas, prevalece el por qué la conservación no puede prescindir de valorización, como de la transformación y de la recualificación, desglosados en la continuación. Las direcciones proyectuales son la conservación, valorización, recualificación y transformación (Paolineli e Valentini, 2009), medidas predominantes y de referencia, respectivamente, de valores patrimoniales a salvaguardar, de permanencias históricas y de áreas con particular valor naturalista para aquellos que tienen niveles distintos de integridad. Las categorías antes mencionadas, pero con mayor grado de fragilidad, con compromisos exigentes de activación de acciones son dirigidas a salvaguardar, contemporáneamente, en función de la valorización del potencial no expresado; los paisajes comprometidos y degradados, son destinados a las nuevas áreas de asentamiento, generalmente sin relación con el contexto pre-existente, ambiental y construido, a usos generalmente de menos importancia; a las categorías citadas anteriormente, en las cuales se observan profundas alteraciones de los ordenamientos históricos y severas deficiencias cualitativas, requieren intervenciones no solamente de recualificación, sino también, de transformación.

En secuencia, para cada unidad de paisaje son establecidas la criticidad, los valores patrimoniales, los objetivos y las direcciones proyectuales; considerando que es posible comparar más de una dirección proyectual en una misma unidad de paisaje. Esta etapa tiene como elemento metodológico de representación el mapeo de la unidad de paisaje con la indicación de las direcciones proyectuales. Para el objeto patrimonial aislado, la propuesta proyectual es presentada por medio de un memorial descriptivo y una representación gráfica (en escala determinada). Las etapas de las actividades están elaboradas para contribuir en la construcción de una base de datos, siguiendo la lógica de elaboración de los Atlas Patrimoniales y sirven para subsidiar intervenciones de mantenimiento y/ o aumentar el valor del objeto de estudio. Para el memorial, se pide la descripción del proceso de trabajo, indicando el procedimiento metodológico adoptado (etapas anteriores), presentación del bien patrimonial a partir de sus valores, caracterización de intervención: restauración física y funcional y/o propuesta de nueva arquitectura. En esta última situación, se debe establecer el principio de la relación entre lo antiguo y lo nuevo (inclusión, intersección o exclusión) y el criterio de intervención (congruencia formal, renovación estilística y figurativa, homologación tipológica); la explicitación de aspectos del entorno urbano adoptados como determinantes en la propuesta, ya sean de carácter físico-histórico, geográfico-ambiental, sociocultural. Por ejemplo: relación morfológica (implementación, visibilidad, escala, volumetria, textura, color) y relación 
funcional (continuidad o ruptura en relación con los usos dominantes); indicación y defensa de uso propuesto, explicitando su carácter de continuidad, discontinuidad y/o complementariedad con respecto al uso original y/o al actual; indicación justificada de las direcciones proyectuales: conservación, valorización, recualificación y transformación. Para la representación se solicitan las siguientes piezas gráficas: implantación; volumetría; planes; elevaciones; secciones; plan de cobertura. Además, se indica el entendimiento del concepto arquitectónico adoptado, como propone Lauro Corona y Carlos Lemos (como citado por Silva, 1983, p. 97):

\section{(...) consecuencia formal de una serie de determinantes, tales como el programa del edificio, la conformación topográfica del terreno, la orientación, el sistema estructural adoptado, las condiciones locales, los fondos disponibles, las condiciones de las posturas que regulan las construcciones y principalmente, la intensión plástica del arquitecto}

Concluyendo, como método, se confecciona un esquema de la trayectoria metodológica adoptada en la disciplina (Figuras 2), con las actividades analíticas, sintéticas y proyectuales y sus respectivos contenidos, subdivididos en etapas de representación y planificación.

Se destaca que, no se propone una estandarización de las herramientas de representación para ser utilizadas por los estudiantes, para no obstaculizar su poder cognitivo y comunicativo. Por lo tanto, la percepción de características peculiares del territorio podrían ser transformadas en una representación de identidad, desvinculada de la cartografía tradicional. El único criterio adoptado es la utilización de base cartográfica del Plan Director Universitario, objetivando proporcionar referencias de escala y de localización geográfica. Desde un punto de vista didáctico, esa estrategia no relaciona el dibujo como método de trabajo, constituyendo asi una actitud reflexivocrítica. Propone también la ausencia de jerarquía entre docente-aprendiz-discente, visto que la construcción de una metodología disciplinar ocurre simultáneamente con la disciplina, de acuerdo con las demandas y las necesidades de los participantes. En este sentido, el experimento busca promover una ruptura reciproca de la modernización de las técnicas, común en la enseñanza actual de arquitectura, cuando en lugar de una herramienta o técnica avanzada de representación y de la enseñanza tradicional, se propone una metodología horizontal e integrada.

\section{Resultados y Discusiones}

Para esa etapa, tal como se describió anteriormente, se obtuvieron como resultado hojas, mapeos y proyectos. Siendo así, se exponen los resultados vinculados a las tres etapas de estructuración de la experiencia. Después de un trabajo de campo, en la etapa de Análisis: Construcción del Marco Cognitivo, se mapea la expresión del proceso, producida sobre proyección de Base Cartográfica integrada al Plano Físico do Campus de Goiabeiras (Anexo 4 - Resolução n 43/2017, p.18) en cuadro blanco. Según COCULTURA (2012) son adoptados los siguientes valores: 1. Valor arquitectónico; 2. Valor de antigüedad; 3. Valor histórico; 4. Valor tecnológico; 5. Valor urbano; 6. Valor asociativo; 7. Valor de autenticidad.

De acuerdo con los debates en clase, son enumerados por los pasantes como fundamentales a catalogación en inventario: 8a. Valor de memoria; 8b. Valor de referencia; 8c. Valor ambiental; 8d. Valor artístico. Esa categorización no ocurre de manera excluyente, por lo tanto, un mismo bien puede tener uno o más valores asignados. Cada estudiante marca en el mapa los valores atribuidos individualmente y produce el debate en clase con las razones de esas atribuciones, y, se da la posibilidad de cancelar la categorización anterior.

El resultado de esa categorización se encuentra en la imagen abajo (Figuras de 3). En síntesis, de 159 bienes construidos mencionados en el Plan Director Universitario, fueran identificados 7 conjuntos y 7 bienes aislados, además de áreas de valor ambiental como manglares, lagunas, formaciones rocosas, ríos, áreas de uso público cultural y circunvalación, consideradas indisociables del análisis.

Junto con la discusión son producidas las hojas de catalogación del preinventario para cada edificio identificado con valor patrimonial, según el modelo de abajo. En este momento, la información sobre el bien se enumera en tres categorias: la primera, sobre las informaciones proyectuales, históricas, contextualización; la segunda, relativo al estado de conservación del bien, para entender si el bien está sometido a algún riesgo inminente, o si está vinculado a algún proceso de 
protección dado por el Plan Maestro o un proyecto de restauración en curso, etc.; por último, se revisa la documentación gráfica disponible sobre la propiedad, como planos, elevaciones, secciones, cartografías, fotografía históricas. Estas sirven para abastecer un sistema de información sobre los edificios, buscando un análisis integrado de todo el conjunto, entendiéndolo de manera diacrónica. En este sentido, la representación crea:

un repertorio visual de morfemas gráficos cuyo entrelazamiento, como en una historia. encuadra la consistencia patrimonial de la estructura territorial, demarca su estado de salud e identifica estrategias para rehabilitar y abrir una nueva etapa de desarrollo coherente del territorio (Magnaghi, 2014, p. 116).

Aún en la proyección cartográfica, los estudiantes definen la dimensión a largo plazo de los edificios levantados y, junto con la percepción dada a través de los estudios de campo y los debates, se definen unidades de paisaje previas, sobre las cuales se definen, otros límites más precisos que son realizadas en las etapas siguientes.

En la segunda etapa, de síntesis: los dados adquiridos en la etapa anterior son confrontados en representaciones específicas, realizadas de acuerdo con las unidades de paisaje divididas anteriormente (Figura 4). Uno de los ejemplos de representación propuesto por los estudiantes es la cualificación de valor (en una escala alta, media y baja), que cruza la información a largo plazo, el valor asignado y la conservación. Esta representación muestra indicios del grado de intervención necesaria, su emergencia, y confiere indicativos acerca de cuál será la teoría de restauración más adecuada. Otra propuesta de los estudiantes segmenta el análisis de las capas físicas y construidas, sugiriendo una jerarquía entre las capas, considerando la larga duración y el grado de degradación/ restauración ambiental. En este sentido, hay un esfuerzo de jerarquizar las capas predominantes del lugar como elemento de valor. Desde esta perspectiva, se percibe la necesidad de una subdivisión de las unidades de paisaje para mejorar la toma decisiones en la tercera etapa, conectando la intervención arquitectónica a la capa física, que es predominante en la unidad.

Sirve como subsidio a la tercera y última etapa, el Escenario Estratégico/ Proyecto, la experimentación, en la cual son enumeradas las direcciones proyectuales de intervención, considerando los puntos críticos y los valores de cada unidad de paisaje; $y$, después de las discusiones hechas en los grupos de trabajo, son definidos los objetivos para cada unidad de paisaje y sus correspondientes direcciones proyectuales. Tales objetivos direccionan la producción de inserciones formales, como "integrar las sub-unidades de paisaje", "unificar y proponer nuevas funcionalidades", "mejorar la inter-relación entre los llenos y vacíos y retirar elementos que interfieran en la integridad de la subunidad", "manutención y recualificación de elementos pre-existentes", resultando en propuestas para los edificios, los espacios abiertos y sus conexiones.

Como ejemplo de esas dos etapas se ilustran los dos procesos de trabajo de la Unidad de Paisaje B (propuesta de los pasantes Daniele Ramos, Júlia Schunck e Mylla Sepulchro) y de la Unidad de Paisaje E (propuesta de los pasantes Juliana Calado, Kamila Salarini e Thabata Coelho) (Figuras de 5 a 7).

\section{Conclusión}

El contenido presentado es efectivamente utilizado, se demuestra en el análisis, la valoración y la lista de intervenciones de acuerdo con la preservación del patrimonio de la Universidade do Espírito Santo, y el proceso en su conjunto genera lecturas, discusiones y debates sobre las decisiones y cuestiones críticas de la actividad y objeto. La construcción de un conocimiento teórico-empírico integrado a la inserción del estudiante en la dinámica de actuación fortalece la relación objeto-sujeto, generando una aproximación y consecuentemente un mayor conocimiento del bien a ser preservado.

Así, al ofrecer el desarrollo de una visión y de conciencia críticas acerca del estado de los bienes de valor y de las posibilidades de intervención a ser realizadas, la disciplina manifiesta el compromiso social del individuo y su papel en la preservación de lo que se pretende se mantener para la posteridad.

Siendo fundamentalmente empírico-cualitativa, la experiencia relatada puede y debe ser objeto de evaluación y alteración, para garantizar su carácter procesal. De 
esta condición, se deriva la posibilidad de ser incluidos otros criterios y parámetros de análisis, así como categorías de descripción e interpretación. Por lo tanto, en función de cada elemento de identificación -grado de conservación, tipología de valor, vulnerabilidad y riesgo- pueden ser atribuidos valores según la criticidad y potencialidad del sitio de aplicación metodológica.

Además, el tema del patrimonio introducido a los estudiantes terminales y en un plan de estudios de 90 (noventa) horas, en una única disciplina, implica una selección rigurosa de criterios que deben adoptarse y que incorporan etapas preponderantes para el proceso, teniendo en cuenta el alcance de la formación de profesionales con capacidad para articular los diversos campos de la Arquitectura y el Urbanismo.

En este sentido, la introducción del estudiante a modelos potenciadores de inmersión en el conocimiento de acuerdo con una combinación de historia, teoría y praxis potencializa el trato de la representación no como herramienta de documentación, sino como un proceso de inmersión cognitiva capaz de revelar cualidades invisibles, o sea, como representación de identidad.

La experimentación teórico-metodológica realizada en la disciplina "Patrimonio Historico, Artístico y Cultural" se constituye como procedimiento operativo capaz de actuar en la formulación de una actitud activa frente al conocimiento, como también en la formación de un pensamiento crítico, frente a los procesos de descualificación, degradación, ruptura e incluso en la pérdida de referencias patrimoniales, en el todo o en parte. En este sentido, al utilizar la tríada temática Patrimonio-EnseñanzaProyecto, la misma contribuye en la formación de profesionales sujetos-colectivos despiertos para una actuación en el territorio, con sensibilidad para hacer crecer vínculos con el lugar. 